\title{
Experience with intraoperative navigation and imaging during endoscopic transnasal spinal approaches to the foramen magnum and odontoid
}

\author{
Omar Choudhri, M.D., ${ }^{1}$ Stefan A. Mindea, M.D., ${ }^{1}$ Abdullah Feroze, B.S., ${ }^{1}$ \\ Ethan Soudry, M.D., ${ }^{2}$ Steven D. Chang, M.D., ${ }^{1}$ and Jayakar V. Nayak, M.D., Ph.D. ${ }^{2}$ \\ Departments of ${ }^{1}$ Neurosurgery and ${ }^{2}$ Otolaryngology-Head \& Neck Surgery, Stanford University Medical \\ Center, Stanford, California
}

Object. In this study the authors share their experience using intraoperative spinal navigation and imaging for endoscopic transnasal approaches to the odontoid in 5 patients undergoing $\mathrm{C} 1-2$ surgery for basilar invagination at Stanford Hospital and Clinics from 2010 to 2013.

Methods. Of these 5 patients undergoing C1-2 surgery for basilar invagination, 4 underwent a 2-tiered anterior C1-2 resection with posterior occipitocervical fusion during a first stage surgery, followed by endoscopic endonasal odontoidectomy in a separate setting. Intraoperative stereotactic navigation was performed using a surgical navigation system in all cases. Navigation accuracy, characterized as target registration error, ranged between $0.8 \mathrm{~mm}$ and 2 $\mathrm{mm}$, with an average of $1.2 \mathrm{~mm}$. Intraoperative imaging using a CT scanner was also performed in 2 patients.

Results. Endoscopic decompression of the brainstem was achieved in all patients, and no intraoperative complications were encountered. All patients were extubated within 24 hours after surgery and were able to swallow within 48 hours. After appropriate initial reconstruction of the defect at the craniocervical junction, no postoperative CSF leakage, arterial injury, or need for reoperation was encountered; 1 patient developed mild postoperative velopharyngeal insufficiency that resolved by the 6-month follow-up evaluation. There were no deaths and no patients required tracheostomy placement. The average inpatient stay after surgery varied between 72 and 96 hours, without extended intensive care unit stays for any patient.

Conclusions. Technologies such as intraoperative CT scanning and merged MRI/CT can provide the surgeon with detailed, virtual real-time information about the extent of complex endoscopic vertebral segment resection and brainstem decompression and lessens the prospect of revision or secondary procedures in this challenging surgical corridor. Moreover, patients experience limited morbidity and can tolerate early oral intake after transnasal endoscopic odontoidectomy. Essential to the successful undertaking of these endoscopic adventures is 1) an understanding of the endoscopic nasal, skull base, and neurovascular anatomy; 2) advanced and extended-length instrumentation including navigation; and 3) a team approach between experienced rhinologists and spine surgeons comfortable with endoscopic skull base techniques

(http://thejns.org/doi/abs/10.3171/2014.1.FOCUS13533)

\begin{abstract}
KEY WoRdS - basilar invagination - craniocervical junction • dens • endoscopic transnasal surgery $\quad$ intraoperative image-guided navigation
\end{abstract}

$\mathrm{T}$ THE craniocervical junction is the highest point of the spinal axis and is often the seat of pathological processes such as rheumatoid disease, degenerative fibroconnective tissue disease, osteomyelitis, and primary or metastatic neoplasms. Lesions in the area of C1-2 can lead to neurovascular compromise due to direct compression and mass effect on the medulla and cervical spinal cord. Operative approaches to the craniocervical junction may involve both anterior and posterior access depending on the location and severity of compression.

Traditionally, the atlas and dens (odontoid process) are accessed anteriorly using a transoral approach. ${ }^{3}$ This approach is still considered the gold standard for addressing high cervical spine pathologies. ${ }^{25}$ More recently, en- doscopic transnasal and transcervical approaches have gained wider acceptance, given the optimized illumination of the craniocervical junction that can be achieved through endoscopy. ${ }^{27,40}$ This approach allows for improved visualization of this anatomically distant surgical field, and more accurate and early recognition of vital neurovascular structures, surgical borders, and lesion margins. The advent of image-guided intraoperative navigation of the cervical spine allows accurate localization of anatomical structures during endoscopic craniocervical junction surgery. This is especially critical for endoscopic spine surgery where the endoscope offers a $2 \mathrm{D}$ view and manipulations by the surgeon are performed at a 10 - to $14-\mathrm{cm}$ distance from the skull base site of in- 
terest. The "intraoperative GPS" provided by navigation for the anterior cervical spine therefore serves as a crucial localization and topographical confirmation tool during these advanced surgical procedures.

Occipitocervical and atlantooccipital joints allow head flexion-extension and head turning, respectively. Significant displacement occurs at these joints from both skin and soft-tissue traction at the neck. Due to the extent of intersegmental movements at the craniocervical junction and lack of superficial bone structures, accurate intraoperative navigation can provide a notable challenge. Improved navigation systems and instrumentation allow reliable navigation at the skull base and craniovertebral junction. Additionally, intraoperative imaging with CT, navigated fluoroscopy, and intraoperative MRI now make endonasal craniocervical surgery a more safe and measured procedure. In this case series, we share our experience using intraoperative spinal navigation and imaging with an endoscopic transnasal approach to the odontoid.

\section{Methods}

\section{Study Population}

We retrospectively evaluated all patients undergoing transnasal endoscopic C1-2 surgery at Stanford University Hospital and Clinics from 2008 to 2013. All surgeries were performed by J. V. N. and S. A. M. We analyzed intraoperative navigation data, imaging completed during the surgeries, patient information, and outcomes. Sources of data were intraoperative records, StealthStation S7 (Medtronic) data, as well as electronic medical records, including inpatient and clinic encounters.

\section{Surgical Technique}

Endoscopic transnasal C1-2 surgery has been described elsewhere by the senior author ${ }^{27}$ but is briefly reviewed here (Fig. 1). Patients are positioned in a Mayfield head holder, and somatosensory evoked potential neuromonitoring is employed. Registration of intraoperative navigation is then performed, which in our series was used universally, with intraoperative CT scanning used in select cases. Preoperative antibiotics are administered before the start of each case. The procedure is performed by an otolaryngologist and a neurosurgeon working in tandem. An endoscope, suction, and irrigation typically enter via the right nostril while the dissecting instruments for skull base surgery, extended-length telescoping drill bits, ultrasonic aspirators, and extended-length flexible and rigid navigation probes enter via the left nostril. An inferior septectomy is performed, removing $2 \mathrm{~cm}$ of vomer bone at its junction with the hard palate. We do not routinely perform sphenoidotomies but rather use the highest point of the posterior slope of the clivus, or the clivus-nasal septum junction, as the superior limits of the dissection. At this point, we note critical anatomical landmarks, namely, the clivus-septum junction superiorly, eustachian tubes laterally, and nasal floor/soft palate inferiorly as marked by the hard and soft palate. The navigation probe is used to identify midline and superoinferior limits of the exposure. Monopolar electrocautery
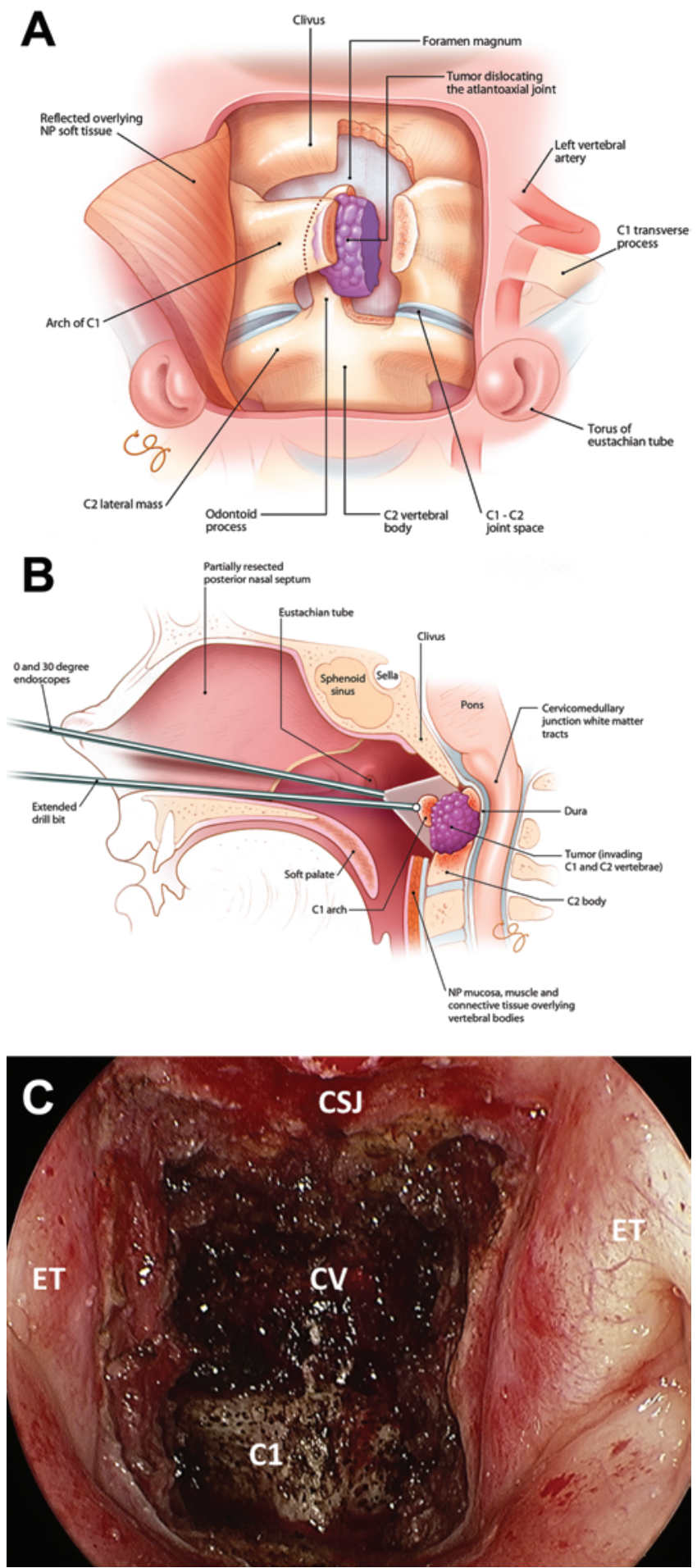

FIG. 1. Images demonstrating the endoscopic transnasal odontoid approach. A: Coronal illustration showing relationships in transnasal odontoid resection. B: Sagittal illustration showing the access angle during a transnasal odontoid approach. C: Endoscopic view of the craniovertebral junction after exposure and before removal of the anterior arch of C-1. Figures adapted with permission from Nayak JV, Mindea SA: Transnasal endoscopic approach to the craniocervical junction. In: Rhinology: Diseases of the Nose, Sinuses, and Skull Base, Kennedy DW and Hwang PH, eds., Thieme, 2012. CSJ = clival septum junction; CV = clivus; $\mathrm{C1}=$ anterior arch of $\mathrm{C}-1$; $\mathrm{ET}=$ eustachian tube; $\mathrm{NP}=$ nasopharynx. 


\section{Navigation and imaging for endoscopic transnasal odontoid surgery}

is then used to resect the nasopharyngeal mucosa with longus colli musculature. Additionally, soft tissue is removed using a microdebrider or ultrasonic aspirator to expose the body and arch of C-1 and C-2. A 3- to 4-mm diamond drill bit along with Kerrison rongeurs is used to remove the anterior arch of $\mathrm{C}-1$. This exposes the $\mathrm{C}-2$ body/dens, which is then drilled at its base to resect the tip and body of the dens from the remaining vertebral ring. Dens removal is typically challenging due to adhesion to the dural lining and osteitis in the setting of disease. The dens may be adherent to the adjacent brainstem dura with admixed fibroconnective soft tissue, such as in rheumatoid pannus, and a CSF leak may be encountered at this stage. In some cases piecemeal removal of the dens may be required. Brainstem decompression is achieved once a wide field of dural pulsations is established. The reconstruction is completed with the layered placement of Gelfoam, fibrin sealant, and bioabsorbable packing material. If a CSF leak is encountered in this distant site, the multilayered closure may also include dural graft substitutes, abdominal fat, and a variety of pedicled regional flaps to reconstruct the defect. A lumbar drain is often placed in the latter situations for 2-3 days to divert CSF.

\section{Intraoperative Navigation and Imaging Protocol}

All patients undergoing extended skull base $\mathrm{C} 1-2$ surgeries complete a single thin-slice sinus and nasopharynx CT scan to delineate bone anatomy such as the hard palate, sphenoid sinus, clivus, and atlantoaxial space (Fig. 2). This high-resolution CT study is completed just prior to the surgery, but after posterior fusion has been performed to account for subtle alterations following plate fixation. Images are then "pushed" from the PACS system to the image-guidance system of interest, or loaded using a CD-ROM or USB external drive. A stereotactic MR image of the brain extending to the upper cervical spine is also completed. Depending on the scenario, a CT angiogram with thin slices $(0.625 \mathrm{~cm})$ can be performed after occipitocervical fusion to delineate skull base vascular anatomy (primarily the vertebral basilar and carotid arteries) that also may undergo small subtle changes in its bone relationships following posterior fusion.

To maintain the most accurate fixed point throughout a lengthy surgery such as this, a temporary skull reference post can be placed for use with both electromagnetic and line-of-sight image guidance systems. Once the patient is intubated and positioned in the Mayfield holder (varies depending on surgeon preference), a scalp incision is made to place a bone post in the post shown. A $2-\mathrm{cm}$ incision is made in the scalp and dissected to the calvaria, the pericranium is cleared completely, and the image guidance post is secured into the bone with self-tapping screws. Once linked with the selected image guidance platform, the post serves as a fixed landmark for the patient that is detected by the navigation system. Patient facial surface landmarks are then used to register to the preexisting soft-tissue landmarks on the 3D volumetric $\mathrm{CT}$ reconstruction performed by the given software platform. On many platforms, the stereotactic registered CT scan can be merged with the stereotactic MR image to allow overlapping views of the bone and soft-tissue al- gorithms from these studies. This technological advancement allows the surgery team to alternate between CT and MRI views exclusively, or hybrid CT/MRI views, to more confidently advance the surgery for challenging skull base sites.

At Stanford, the CereTom intraoperative CT scanner (Neurologica Corporation) is brought into the surgical field in select cases and positioned around the patient's head while the patient is intubated. All intraoperative scanner devices use appropriately sized head holders that allow easy positioning of the intraoperative scanner during any part of the resection. Appropriate radiation precautions need to be enforced during the performance of the CT scan and images can be rapidly reconstructed to assess the extent of skull base bone resection.

\section{Results}

A total of 5 patients underwent transnasal endoscopic C1-2 surgery with spinal navigation (Table 1). One patient was male and 4 were female, and the age range was 5587 years (mean 67 years). Of these 5 patients, 4 underwent resection of $\mathrm{C} 1-2$ while 1 patient underwent biopsy only. The underlying cause of C-2 disease was osteomyelitis (2 patients), rheumatoid arthritis (2 patients), and metastatic breast cancer (1 patient). All patients undergoing $\mathrm{C1}-2$ resection underwent a 2-stage surgery with a primary occipitocervical fusion, followed by endoscopic endonasal odontoidectomy during the second stage. The time period between stages varied from 3 days to 10 weeks based on individual patient parameters. Stereotactic navigation with Stealth S7 or Fusion (Medtronic) platforms was used in all cases. Navigation accuracy, characterized as target registration error, ranged between $0.8 \mathrm{~mm}$ and $2 \mathrm{~mm}$ (mean $1.2 \mathrm{~mm}$ ). Intraoperative imaging using the CereTom CT scanner was completed in 2 patients. All patients underwent successful brainstem decompression with odontoid resection (4 patients) or biopsy (1 patient), and no intraoperative complications were encountered. All patients were extubated within 24 hours after surgery and were able to swallow independently with good oral intake within 48 hours after surgery. No postoperative CSF leakage or arterial injury was encountered; 1 patient developed mild postoperative velopalatal insufficiency that improved by the 6-month follow-up evaluation. There were no deaths in this series and none of the patients required a tracheostomy. The average inpatient stay after surgery varied between 72 and 96 hours.

\section{Illustrated Cases}

\section{Case 1}

A 55-year-old man with a history of diabetes, hypertension, and intravenous drug abuse presented with 2 months of progressive headache and neck pain, along with difficulty walking, intermittent fevers, and fatigue. He was found to have elevated acute phase reactants and had a history of remote anterior cervical surgery in the past. A clinical examination revealed normal strength in the upper and lower extremities with evidence of hyperre- 

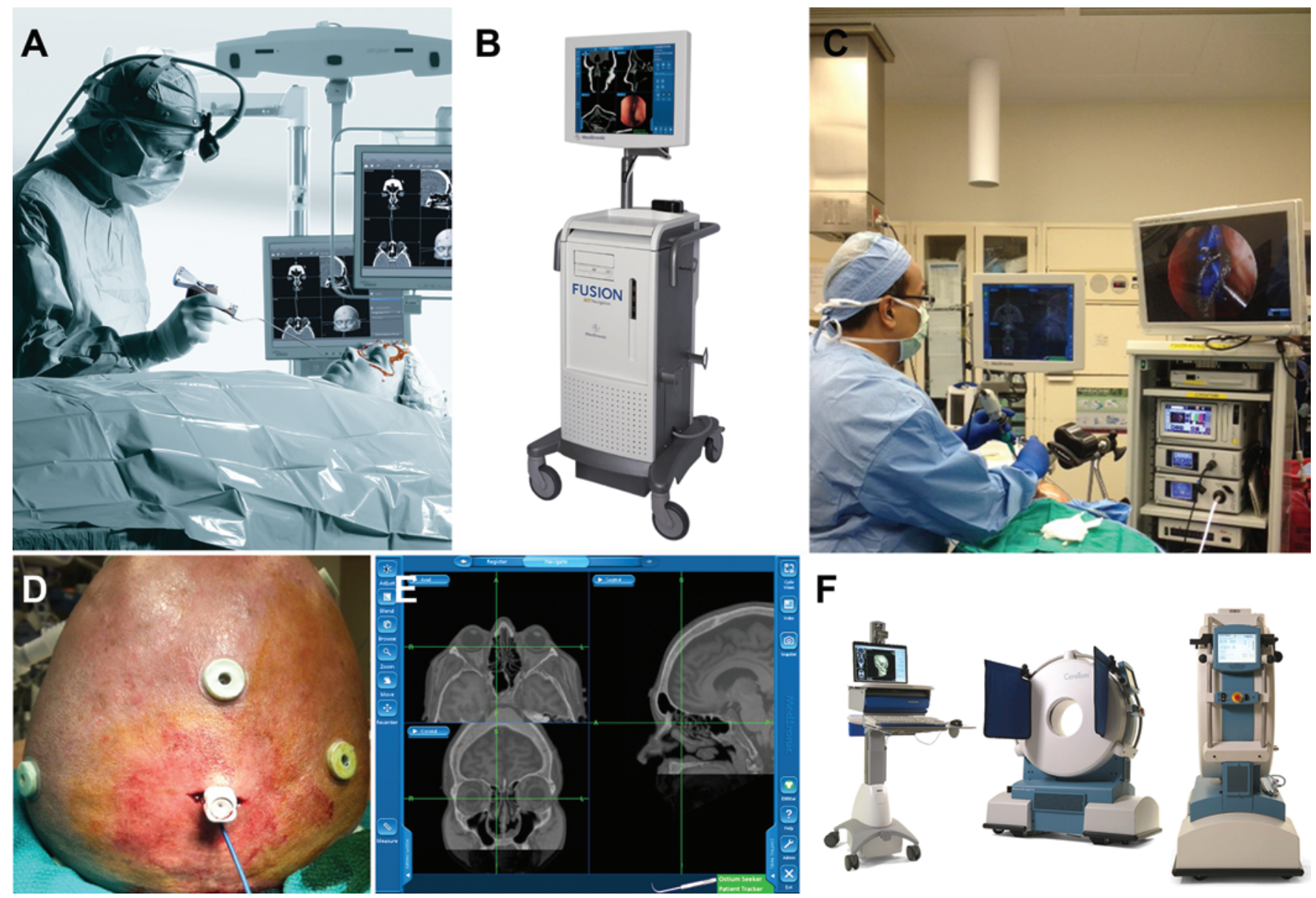

FIG. 2. Navigation and imaging tools for transnasal odontoid resection. A: Stryker Optical Image Guidance System. Courtesy of Stryker, Inc. B: Medtronic Fusion Navigation. Courtesy of Medtronic, Inc. C: Operating room setup with the electromagnetic field generator for navigation-assisted endoscopic odontoidectomy. D: Skull navigation reference post secured to calvaria along with scalp fiducials to allow accurate registration and the ability to use navigated instruments in the fusion system. E: Fusion navigation system display with a merged CT scan and an MR image to allow simultaneous appreciation of bone and soft-tissue landmarks. F: CereTom intraoperative CT scanner and associated workstation. Courtesy of Neurologica, Inc.

flexia. An MR image of the brain and cervical spine was completed showing soft tissue enhancement and phlegmon formation at $\mathrm{C} 1-2$ with evidence of basilar invagination and brainstem compression (Fig. 3A and B). The patient was started on broad-spectrum antibiotics, and given the instability and impending brainstem compression, decompression and stabilization at the craniocervical junction were advocated. An occiput-C5 fusion was performed at the first stage and was well tolerated. Locally harvested autograft was augmented with morcellized allograft and placed along the instrumentation construct.

Four days later, second-stage endoscopic endonasal C1-2 decompression was performed with resection of the dens infected by osteomyelitis. The dens was particularly challenging to resect in this case given its slightly inferior position in relation to an imaginary line extending posteriorly from the hard palate (Fig. 3C). However, given the patient's thick tongue and dense soft palate, we believed that the transnasal approach was the better option for this patient and did not displace or dissect any normal oral, lip, or palatal structures. We achieved 0.8-mm accuracy using Stealth navigation, which allowed us to safely re- sect the odontoid and associated exuberant inflammatory soft tissue. An intraoperative CereTom CT scan was completed that confirmed complete resection of the odontoid with appropriate brainstem decompression (Fig. 3D). A small intraoperative CSF leak was noted, and thus skull base reconstruction was completed with layered abdominal fat graft, Tisseel fibrin sealant (Baxter Healthcare Corp.), and Gelfoam (Pharmacia). The patient was kept intubated postoperatively and taken to the intensive care unit, where he was extubated uneventfully the next day. He could swallow liquids on the second postoperative day, and a 48-hour CSF diversion was completed and the lumbar drain was removed after a successful clamping trial. On the fourth postoperative day, the patient was discharged home at his baseline neurological level (Fig. 3E).

\section{Case 5}

An 87-year-old woman with a long-standing history of rheumatoid arthritis presented to the hospital after a fall at home with features of spasticity and neck pain on examination. Magnetic resonance imaging of the cervical spine revealed a large C-2 pannus causing basilar 


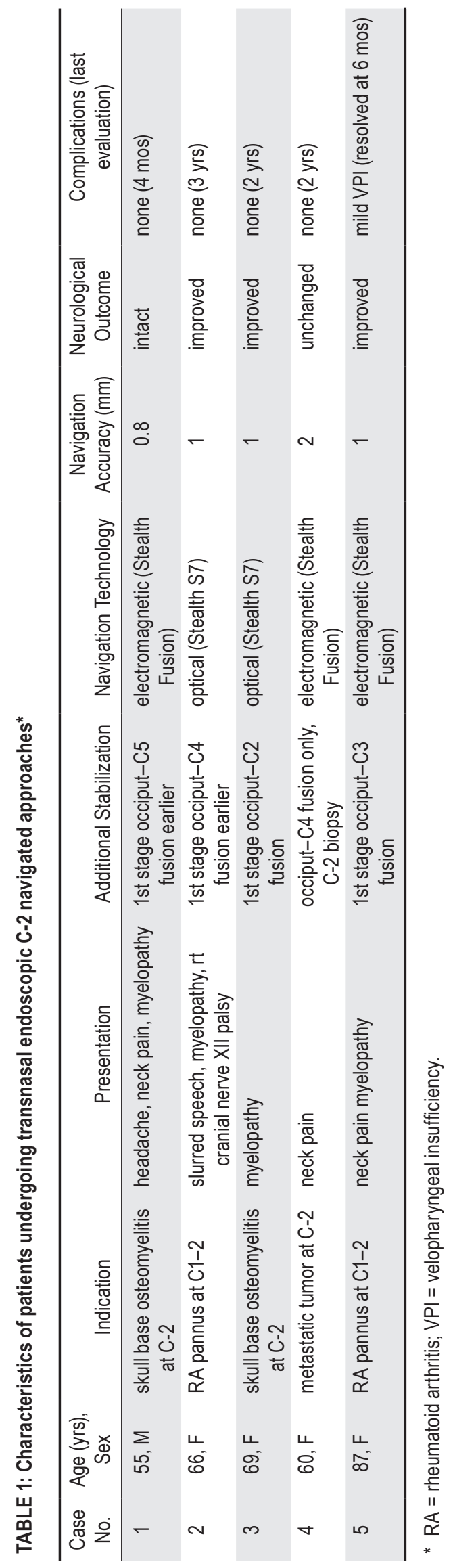

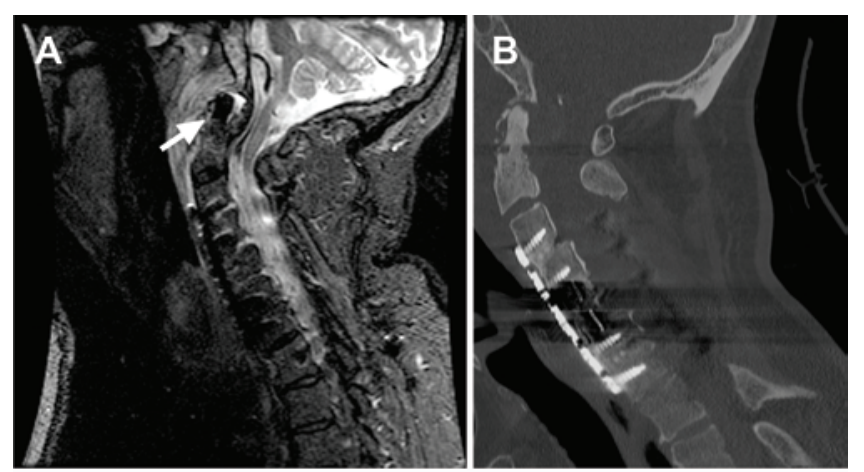
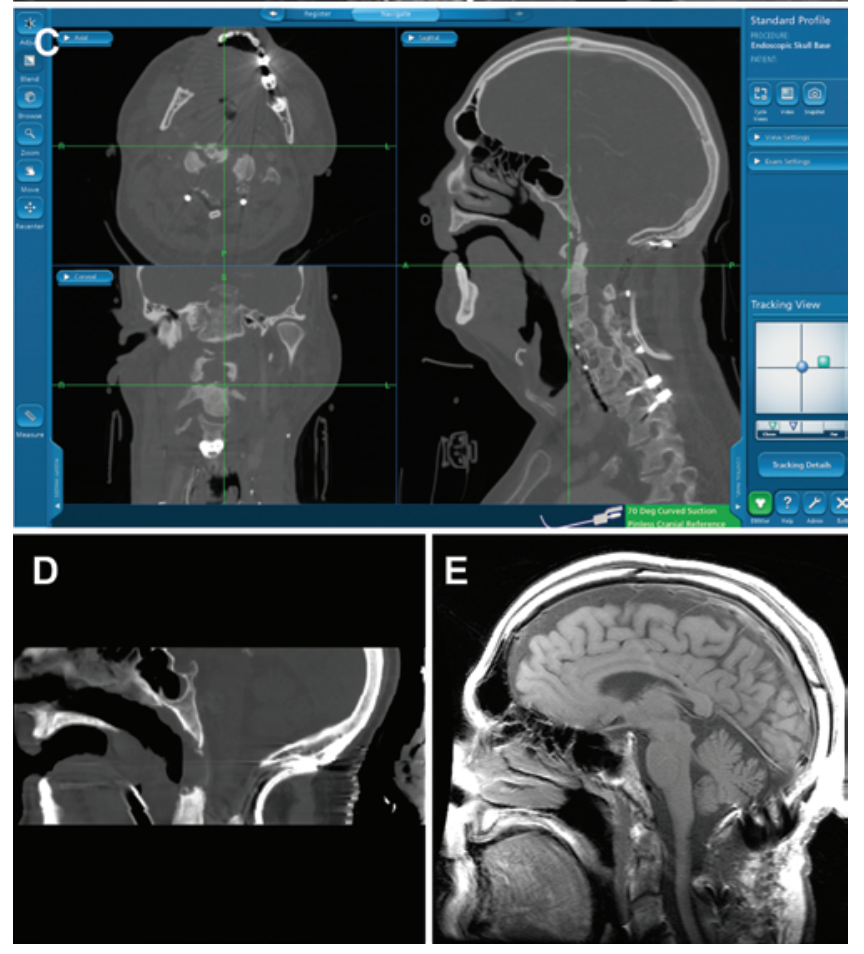

FIG. 3. Case 1. A: Preoperative sagittal STIR sequence MR image demonstrating osteomyelitis at the $\mathrm{C}-2$ dens (arrow) with associated mass effect on the spinal cord with signal change. B: Preoperative sagittal CT scan showing bone destruction of the odontoid caused by infection. Anterior cervical instrumentation hardware from prior surgery is also noted. C: Intraoperative navigation display with a triplanar demonstration of the inferior limit of access obtained through a transnasal approach, with the green cross-hairs indicating that the tip of the navigation probe was contacting the anterior face of the dens. D: Intraoperative sagittal reconstruction of a CereTom CT scan confirming adequate decompression with complete removal of the anterior arch of C-1, infected phlegmon, and odontoid tip. E: Six-month postoperative sagittal T1-weighted MR image showing a decompressed craniocervical junction.

impression, severe cervicomedullary compression, and signal change in the spinal cord (Fig. 4A). Due to concern for severe cord compression, the patient underwent a 2-stage procedure. During the first stage an occiput-C3 fusion was completed using both allograft and autograft bone. The patient tolerated the procedure well and an endoscopic transnasal resection of the odontoid was completed 3 days later. We used stealth navigation with fixed skull post placement and achieved 1-mm accuracy. An uneventful resection of the anterior arch of C-1 was com- 


\section{O. Choudhri et al.}
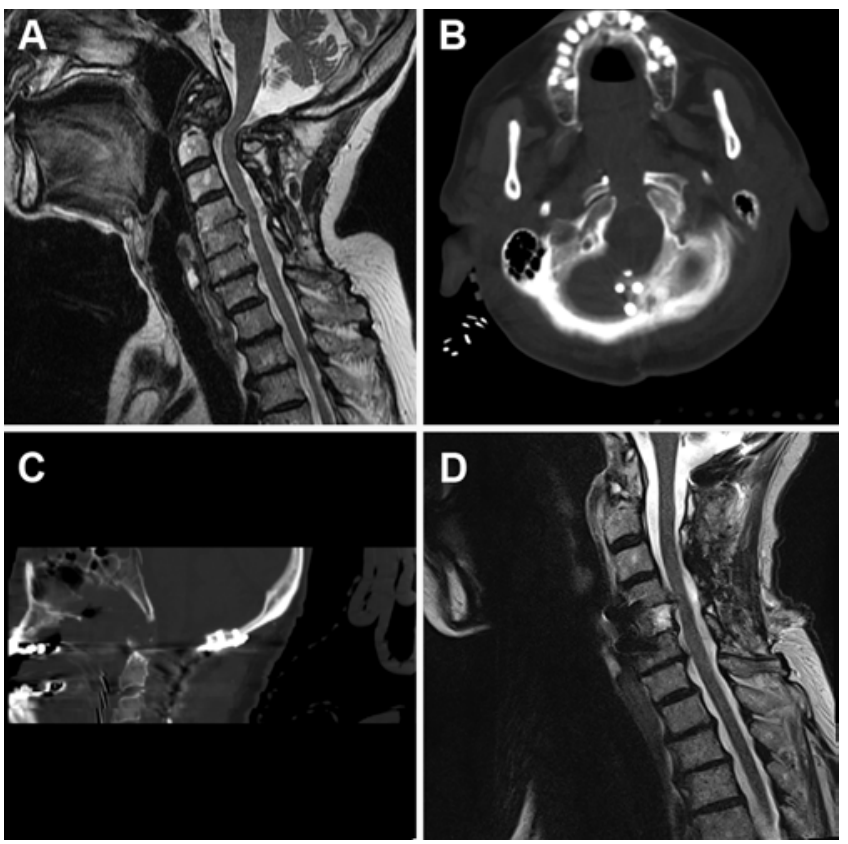

FIG. 4. Case 5. A: Preoperative sagittal T2-weighted MRI sequence with a rheumatoid pannus at the craniocervical junction causing mass effect at the cervicomedullary junction. B: Intraoperative axial CereTom CT scan demonstrating removal of the anterior arch of C-1. C: Intraoperative sagittal CereTom CT scan reconstruction showing adequate decompression with removal of the anterior arch of $\mathrm{C}-1$, rheumatoid pannus, and the displaced odontoid tip. D: Postoperative sagittal T2-weighted MR image demonstrating a completely decompressed cervicomedullary junction.

pleted and partial C-2 corpectomy with removal of an associated rheumatoid pannus was completed. An intraoperative CereTom CT scan demonstrated complete resection of the C-1 anterior arch and C-2 dens, and confirmed adequate decompression of the brainstem (Fig. 4B and C). The patient was extubated within 24 hours of the procedure and tolerated a clear liquid diet at 24 hours after the procedure. She complained of mild swallowing difficulty after the procedure, which was diagnosed as mild velopharyngeal insufficiency after endoscopy. No lumbar drainage was used and she was discharged to a rehabilitation facility on the third postoperative day (Fig. 4D).

\section{Discussion}

Cranial neurosurgical procedures were the first to use stereotactic navigation as early as the 1940s using a frame-based device described by Spiegel and Wycis. ${ }^{29}$ The presence of a cumbersome frame to the patient's head proved to be an impediment to surgical access. This led to the development of frameless stereotactic navigation in the 1970s with early work from Roberts et al. that allowed its incorporation into the surgical microscope. ${ }^{31}$ Initially, frameless stereotactic navigation was used only for superficial lesions, but improvements in CT and MRI technologies improved its accuracy to 2-4 $\mathrm{mm}$. Stereotactic navigation during spine surgery was fraught with inaccuracies due to the lack of stable superficial bone landmarks. Roessler et al. attained a mean application accuracy of $11.3 \mathrm{~mm}$ in the early use of frameless stereotactic navigation for spine surgery. ${ }^{32}$ Intraoperative Carm radiographs are the most common tool used by spine surgeons for assessing anatomy and instrumentation. The development of stereotactic navigation was encouraged as way to reduce radiation exposure during spine surgery. The ISG wand (ISG technologies) was the earliest commercially available stereotactic navigation probe, which was adopted early for surgery around the craniocervical junction. ${ }^{11}$

Given the small operative corridor, the surgeon's distance from the operative site, and the distorted anatomy from disease at the craniocervical junction, the benefits of accurate intraoperative image guidance of direct feedback to the surgeon are immediately realized. Pollack et al. reported the first use of stereotactic navigation for anterior cervicomedullary decompression in which the ISG wand system was used for CT-guided navigation. ${ }^{30}$ The same year, Dyer et al. published their experience with the ISG wand for a transmaxillary odontoidectomy in a patient who could not tolerate a transoral procedure. They used Allegro image processing software, which allowed reconstruction on acquired images in 3D planes. ${ }^{11}$

Transoral odontoidectomy is still considered the gold standard approach to the cervicomedullary junction. It was first described in the 1930s in dog experiments and subsequently described in multiple reports. ${ }^{16}$ Crockard was the first to describe the present-day transoral approach to the upper cervical spine. ${ }^{7}$ Spetzler and colleagues devised the retractor system used for the transoral approach and described their experience in 29 patients, with only $3 \%$ morbidity and no mortality. ${ }^{36}$ In a similar series of 53 patients, Hadley et al. performed a transoral odontoidectomy in which no deaths and a morbidity rate of $6 \%$ occurred. They used microscopy and intraoperative radiographs with a marking instrument in the field or contrast material to assess the depth of exposure. ${ }^{17}$ Dickman and colleagues are further credited with studying the biomechanical effects of transoral odontoidectomy and underscoring the importance of additional spinal stabilization. ${ }^{10}$ Transoral exposure has since been used for multiple occipitocervical pathologies including basilar invagination, rheumatoid disease, fractures, tumor, and odontoid disease. Frempong-Boadu et al. described an endoscopic-assisted transoral approach to the craniovertebral junction in their series of 7 patients and established that this approach improved visualization with a lesser need for palatal splitting and maxillotomy. ${ }^{13}$ While endoscopes improve visualization, use of a transoral approach requires awareness of important considerations. These considerations include difficulty with surgeon access to the skull base given patient dentition, velopharyngeal insufficiency with hypernasal speech, meningitis from bacterial flora in the oral cavity, upper airway/pharyngeal swelling requiring prolonged intubation, dysphagia, and dental and palatal injury. ${ }^{27}$ Additionally, a transoral approach may require extended nasogastric tube feeding to allow oropharynx healing, thus resulting in a longer recovery. The transoral route is difficult to accomplish in patients with micrognathia. ${ }^{23}$

The transcervical approach with a combination of tu- 


\section{Navigation and imaging for endoscopic transnasal odontoid surgery}

bular retraction, frameless stereotaxy, and endoscopy has been successfully used for odontoid resection. ${ }^{20,40}$ An extraoral prevascular approach can provide an even wider angle of approach to the periclival and subaxial area in comparison with the transoral approach, as described by Agrawal et al. in a cadaveric study. ${ }^{1}$ The sublabial route with palatal resection is another approach for anterior brainstem decompression and can provide a wide operative corridor, especially in patients with distorted anatomy. ${ }^{5}$

Alfieri et al. described a "trans-nasal" endoscopic approach to the craniocervical junction in a cadaveric model and identified C-2 as the caudal limit of exposure. ${ }^{2,26}$ Kassam et al. then described the first case of an endoscopic endonasal odontoid resection in a 73-year-old woman with rheumatoid arthritis. ${ }^{22}$ Nayak et al. further corroborated these data in the largest published series of endoscopic endonasal odontoidectomies in patients with rheumatoid arthritis from the same center. ${ }^{27}$ This early experience demonstrated limited morbidity compared with transoral approaches; 2 patients required a tracheostomy due to preoperative pharyngeal dysfunction, and no postoperative CSF leaks or infections occurred. No vascular injuries or procedural deaths were encountered. A concern with transnasal approaches is the anatomical risk of injury to the eustachian tubes, carotid artery, and vidian nerve, which are avoided in transoral routes. These theoretical concerns about neurovascular injury have not been observed in any of the reported transnasal endoscopic odontoid approaches available in the litera-

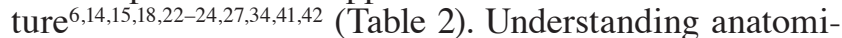
cal relationships is crucial with an endonasal approach; hence, the importance of superior endoscopic visualization and intraoperative image guidance. Of 25 reported cases of endonasal endoscopic odontoid resection thus far, only 1 had a postoperative CSF leak, and no infections were encountered. ${ }^{42}$ In 1 series, a single death was reported in which the patient died later from pulmonary embolus. ${ }^{27}$
A current limitation of endoscopic skull base surgery is $2 \mathrm{D}$ representation of a complex $3 \mathrm{D}$ space, which frequently results in spherical aberrations with "fish bowl" effects. Even though endoscopy allows excellent illuminated visualization, one may lose a sense of depth with endoscopic procedures. Intraoperative navigation complements endoscopic technology well and provides a solution to the intrinsic limitation of spinal/endonasal endoscopy. The image guidance systems for craniocervical surgery employ electromagnetic or optical tracking systems. The Medtronic StealthStation and Fusion systems are the two portals available from Medtronic. In the Fusion image-guided system, an electromagnetic field generator is placed in close proximity to the patient (Fig. 2) while a skull post attached at the start of the case maintains navigation accuracy despite head movement during surgery. A similar setup can be used with the optical tracking system in which a mobile navigation arm recognizes a fixed reference point on the patient or a nearby reference array, as well as reflective spheres to maintain accuracy during surgery. In some cases a post with navigated spheres can be screwed onto the skull before the start of the surgery. We have found the electromagnetic tracking system to have accuracy between $0.8 \mathrm{~mm}$ and $2 \mathrm{~mm}$ during surgery without necessarily requiring the patient's head to be in a halo or Mayfield holder. The BrainLab VectorVision Neuronavigation system has a similar portal for optical tracking and has the advantages of allowing the surgeon to register the end of the endoscope, suction tips, and micro instruments. Ugur et al. used this system for image guidance in their 2 cases of transoral treatment of basilar invagination. ${ }^{37}$ This system can assist with sequential and updated image guidance without the need to switch to a navigation probe. The Iso- $\mathrm{C}$ navigation system, in conjunction with BrainLab image guidance, can allow stereotactic navigation as well as fluoroscopic 3D reconstructions for intraoperative imaging to confirm the extent of resection. Its utility was demonstrated by Laufer

TABLE 2: Reports of navigation techniques used in 29 patients undergoing transnasal endoscopic odontoid approaches*

\begin{tabular}{lcclll}
\hline \multicolumn{1}{c}{ Authors \& Year } & $\begin{array}{c}\text { No. of } \\
\text { Patients }\end{array}$ & $\begin{array}{c}\text { Mean Age } \\
\text { (yrs) }\end{array}$ & Navigation Portal/Imaging Type & $\begin{array}{c}\text { Posterior } \\
\text { Stabilization }\end{array}$ & $\begin{array}{c}\text { Intraoperative } \\
\text { Imaging }\end{array}$ \\
\hline Kassam et al., 2005 & 1 & 73 & Stryker image guidance/CT & yes & none \\
Nayak et al., 2007 & 9 & 76 & used, not mentioned/NA & yes (8/9) & none \\
Wu et al., 2008 & 3 & 44 & BrainLab Vector vision/MRI & yes & none \\
Laufer et al., 2008 & 1 & 25 & BrainLab/CT & yes & yes (Iso-C system) \\
Leng et al., 2009 & 1 & 50 & BrainLab/NA & yes & none \\
Gempt et al., 2010 & 1 & 64 & BrainLab/CT & yes (prior) & none \\
Hankinson et al., 2010 & 2 & 13 & used, not mentioned/NA & yes & none \\
Scholtes et al., 2011 & 1 & 54 & used, not mentioned/NA & no & none \\
Cornelius et al., 2011 & 1 & 57 & Medtronic Stealth/CT & yes & none \\
Grammatica et al., 2011 & 1 & 24 & not mentioned & yes (2/3) & none \\
Yu et al., 2013 & 3 & 51 & used, not mentioned/CT \& MRI & yes (4/5) & yes (CereTom CT \\
current study & 5 & 67 & Medtronic Stealth \& Fusion/CT \& MRI & & in 2 patients) \\
\hline
\end{tabular}

* NA $=$ not available. 


\section{O. Choudhri et al.}

et al. in transnasal endoscopic odontoid resection in a nonachondroplastic dwarf with severe basilar invagination. ${ }^{23}$ Image guidance is equally important in transoral approaches to the cervical spine, where it can provide accuracy with reduced radiation exposure..$^{37,38}$

We have found the combined use of CT and MR technology to be extremely useful for odontoid surgery. This combination allows excellent visualization of bone landmarks as well as soft tissue during endoscopic surgery. A thin-slice CT angiogram $(0.625 \mathrm{~cm} / \mathrm{slice})$ may also be used in combination with the merge application to allow better vascular detail. This angiogram is critical for avoiding the carotid arteries, which are present just anterior and lateral to the $\mathrm{C}-1$ arch and vertebral arteries and which could be as much as $1.1 \mathrm{~cm}$ from the midline at the level of $\mathrm{C}-1$. Leng et al. described the utility of using a preoperative CT angiogram uploaded to the BrainLab system to allow intraoperative identification of vascular anatomy. ${ }^{24} \mathrm{Neo}$ et al. described a case in which a CT angiography-MRI merge with navigation was found to be useful during resection of a skull base chordoma that encased the vertebral artery. ${ }^{28}$

Performance of intraoperative imaging during odontoid surgery can be useful in identifying the extent of bone resection and spinal decompression achieved to that point in the surgery. The endoscopic view can often be disorienting and a suboptimal decompression may result if no intraoperative imaging is attempted. Traditionally, radiographs were obtained to document the extent of bone removal, but soft-tissue discrimination is not present and hence it may not definitely show the extent of decompression. A number of portable CT scanners such as CereTom, Tomoscan (Philips), xCAT (Xoran), and OTOscan (Neurologica) are available that provide images of diagnostic quality and can be accommodated in the operating room setting. ${ }^{33}$ We used a CereTom CT scanner in 2 cases in our series after the majority of resection had been completed to assess if further bone or soft-tissue decompression was required. Difficulty may be encountered in accommodating the patient's head in the scanner gantry if the head is in a Mayfield clamp, in addition to scatter artifact from metal pin placement. We therefore found that the electromagnetic navigation system with a custom headrest was an ideal solution for completing multiple scans during resection without altering navigational accuracy. There are reports on using intraoperative MRI for resection of a cervicomedullary lesion. Kaibara et al. used intraoperative MRI in 3 patients with cervicomedullary lesions using a transoral approach. ${ }^{21}$ Later work by Dhaliwal et al. demonstrated intraoperative MRI to be useful for determining the adequacy of resection, confirming regional anatomy, and determining lesion borders. ${ }^{9}$ The critical role of intraoperative imaging is especially realized if navigation fails or confers suboptimal accuracy. Hickman et al. reported the first case of a pediatric transnasal odontoidectomy requiring a reoperation. An incomplete resection was noted on a postoperative $\mathrm{CT}$ scan, but not intraoperatively due to the loss of stereotactic navigation accuracy secondary to loosened Mayfield pins..$^{19}$

Posterior stabilization is critical in patients undergoing odontoid resection, and we performed an occipitocervical fusion in all of our patients as the first stage operation. No fusion was performed in 1 patient who underwent a transnasal biopsy without extended resection. A critical component of navigation accuracy at the craniovertebral junction is absence of movement at the occipitocervical and atlantoaxial joints. This movement can be mostly eliminated by Mayfield fixation, but having completed a prior occipitocervical fusion ensures absence of these movements. ${ }^{28}$ Cervical fusion therefore allows improved accuracy during endonasal odontoid resection as the upper cervical spine becomes stabilized with the skull base. The sequence of these surgeries is critical and best serves the surgeon in terms of operative ease, accuracy, patient comfort, and improved reduction of craniovertebral deformity. Transoral surgery, however, requires a flexed head position, and thus most patients cannot undergo posterior fusion as the first-stage operation as it would preclude appropriate positioning for the transoral procedure.

The placement of a registration frame is a known issue with craniocervical navigation systems. The reference arc tends to move during surgery and could be problematic. Similarly, fiducial skin markers on the head may be lost with skin shifts during positioning. Occlusal custom-made splints placed into the teeth have been used as fixed reference points by some centers during transoral navigated approaches. ${ }^{39}$ We prefer the use of boneintegrated reference posts screwed into the calvaria with self-tapping screws. This setup, in conjunction with our image-guided navigation system, does not require a reference arc and maintains accuracy with slight movements of the head as long as it is placed within the magnetic field or line of sight. ${ }^{4,35}$ There are few risks with use of the skull post, but they include CSF leakage if the screws are placed over thin calvaria, unsightly scar formation if placed outside of the hairline, and hair follicle loss.

The transnasal craniocervical approach has some inherent limitations. The hard palate restricts access to the subaxial spine, and approaching lesions below C-2 is nearly impossible. ${ }^{19}$ Feasibility of the transnasal C-2 approach is determined by the palatine line, which is "drawn along the floor of the nasal cavity extending from the premaxilla parallel to the hard palate through the craniocervical junction." 12 The palatine line and the nasopalatine line help define the inferior limit of the endonasal approach in this small operative corridor., ${ }^{8,12} \mathrm{Cra}-$ niocervical anatomy can be variable, and patients with platybasia usually have surgical targets above the palatine line. Conversely, a combination of transoral, sublabial, or transcervical approaches may be used in patients with pathologies below the palatine line. ${ }^{5}$

This report represents the second largest series of the transnasal endoscopic spinal approach to the craniocervical junction and the only report with 2 cases of cervicomedullary compression from skull base osteomyelitis. We attempt in this paper to highlight the challenges and share our experience with navigation and intraoperative imaging with transnasal spinal approaches at our center.

\section{Conclusions}

A transnasal endoscopic approach to the $\mathrm{C} 1-2$ area 


\section{Navigation and imaging for endoscopic transnasal odontoid surgery}

is a safe and effective strategy to address craniocervical pathology. It is most effective when used in conjunction with intraoperative frameless electromagnetic stereotactic navigation and, for most cases, should be preceded by occipitocervical stabilization. With intraoperative CT scanning and MRI, the surgeon can determine with confidence the extent of resection and minimize the need for repeat surgeries for incomplete skull base decompression. Moreover, patients are extubated faster and tolerate an oral diet earlier compared with traditional transoral approaches. An understanding of nasopharyngeal and neurovascular anatomy in this region of the skull base is crucial, and a team approach between experienced rhinologists and spine surgeons comfortable with endoscopic skull base techniques is essential for successful outcomes.

\section{Acknowledgments}

We thank Cindy H. Samos for help in preparing the manuscript and Elizabeth H. Hoyte for help in preparing the figures.

\section{Disclosure}

Dr. Nayak serves as a consultant to Medtronic, Stryker Navigation, and Olympus, and is on the Scientific Medical Advisory Board of Polyganics, Inc.

Author contributions to the study and manuscript preparation include the following. Conception and design: Nayak, Choudhri, Mindea. Acquisition of data: all authors. Analysis and interpretation of data: Nayak, Choudhri, Mindea, Feroze. Drafting the article: Choudhri. Critically revising the article: Nayak, Choudhri. Reviewed submitted version of manuscript: Nayak, Choudhri, Soudry, Chang.

\section{References}

1. Agrawal A, Cavalcanti DD, Garcia-Gonzalez U, Chang SW, Crawford NR, Sonntag VKH, et al: Comparison of extraoral and transoral approaches to the craniocervical junction: morphometric and quantitative analysis. World Neurosurg 74: $178-188,2010$

2. Alfieri A, Jho HD, Tschabitscher M: Endoscopic endonasal approach to the ventral cranio-cervical junction: anatomical study. Acta Neurochir (Wien) 144:219-225, 2002

3. Apuzzo ML, Weiss MH, Heiden JS: Transoral exposure of the atlantoaxial region. Neurosurgery 3:201-207, 1978

4. Bernardeschi D, Nguyen Y, Villepelet A, Ferrary E, Mazalaigue $S$, Kalamarides $M$, et al: Use of bone anchoring device in electromagnetic computer-assisted navigation in lateral skull base surgery. Acta Otolaryngol 133:1047-1052, 2013

5. Bettegowda C, Shajari M, Suk I, Simmons OP, Gokaslan ZL, Wolinsky JP: Sublabial approach for the treatment of symptomatic basilar impression in a patient with Klippel-Feil syndrome. Neurosurgery 69 (1 Suppl Operative):ons77-ons82, 2011

6. Cornelius JF, Kania R, Bostelmann R, Herman P, George B: Transnasal endoscopic odontoidectomy after occipito-cervical fusion during the same operative setting-technical note. Neurosurg Rev 34:115-121, 2011

7. Crockard HA: The transoral approach to the base of the brain and upper cervical cord. Ann R Coll Surg Engl 67:321-325, 1985

8. de Almeida JR, Zanation AM, Snyderman CH, Carrau RL, Prevedello DM, Gardner PA, et al: Defining the nasopalatine line: the limit for endonasal surgery of the spine. Laryngoscope 119:239-244, 2009

9. Dhaliwal PP, Hurlbert RJ, Sutherland GS: Intraoperative magnetic resonance imaging and neuronavigation for transoral ap- proaches to upper cervical pathology. World Neurosurg 78: $164-169,2012$

10. Dickman CA, Crawford NR, Brantley AG, Sonntag VKH: Biomechanical effects of transoral odontoidectomy. Neurosurgery 36:11461153, 1995

11. Dyer PV, Patel N, Pell GM, Cummins B, Sandeman DR: The ISG viewing wand: an application to atlanto-axial cervical surgery using the Le Fort I maxillary osteotomy. Br J Oral Maxillofac Surg 33:370-374, 1995

12. El-Sayed IH, Wu JC, Dhillon N, Ames CP, Mummaneni P: The importance of platybasia and the palatine line in patient selection for endonasal surgery of the craniocervical junction: a radiographic study of 12 patients. World Neurosurg 76:183-188, 2011

13. Frempong-Boadu AK, Faunce WA, Fessler RG: Endoscopically assisted transoral-transpharyngeal approach to the craniovertebral junction. Neurosurgery 51 (5 Suppl):S60-S66, 2002

14. Gempt J, Lehmberg J, Meyer B, Stoffel M: Endoscopic transnasal resection of the odontoid in a patient with severe brainstem compression. Acta Neurochir (Wien) 152:559-560, 2010

15. Grammatica A, Bonali M, Ruscitti F, Marchioni D, Pinna G, Cunsolo EM, et al: Transnasal endoscopic removal of malformation of the odontoid process in a patient with type I ArnoldChiari malformation: a case report. Acta Otorhinolaryngol Ital 31:248-252, 2011

16. Greenberg AD, Scoville WB, Davey LM: Transoral decompression of atlanto-axial dislocation due to odontoid hypoplasia. Report of two cases. J Neurosurg 28:266-269, 1968

17. Hadley MN, Spetzler RF, Sonntag VKH: The transoral approach to the superior cervical spine. A review of 53 cases of extradural cervicomedullary compression. J Neurosurg 71:16-23, 1989

18. Hankinson TC, Grunstein E, Gardner P, Spinks TJ, Anderson RC: Transnasal odontoid resection followed by posterior decompression and occipitocervical fusion in children with Chiari malformation Type I and ventral brainstem compression. Report of 2 cases. J Neurosurg Pediatr 5:549-553, 2010

19. Hickman ZL, McDowell MM, Barton SM, Sussman ES, Grunstein E, Anderson RC: Transnasal endoscopic approach to the pediatric craniovertebral junction and rostral cervical spine: case series and literature review. Neurosurg Focus 35(2):E14, 2013

20. Hsu W, Kosztowski TA, Zaidi HA, Gokaslan ZL, Wolinsky JP: Image-guided, endoscopic, transcervical resection of cervical chordoma. Technical note. J Neurosurg Spine 12:431435,2010

21. Kaibara T, Hurlbert RJ, Sutherland GR: Intraoperative magnetic resonance imaging-augmented transoral resection of axial disease. Neurosurg Focus 10(2):E4, 2001

22. Kassam AB, Snyderman C, Gardner P, Carrau R, Spiro R: The expanded endonasal approach: a fully endoscopic transnasal approach and resection of the odontoid process: technical case report. Neurosurgery 57:E213, 2005

23. Laufer I, Greenfield JP, Anand VK, Härtl R, Schwartz TH: Endonasal endoscopic resection of the odontoid process in a nonachondroplastic dwarf with juvenile rheumatoid arthritis: feasibility of the approach and utility of the intraoperative Iso-C three-dimensional navigation. Case report. J Neurosurg Spine 8:376-380, 2008

24. Leng LZ, Anand VK, Hartl R, Schwartz TH: Endonasal endoscopic resection of an os odontoideum to decompress the cervicomedullary junction: a minimal access surgical technique. Spine (Phila Pa 1976) 34:E139-E143, 2009

25. Menezes AH: Surgical approaches: postoperative care and complications "transoral-transpalatopharyngeal approach to the craniocervical junction." Childs Nerv Syst 24:1187-1193, 2008

26. Messina A, Bruno MC, Decq P, Coste A, Cavallo LM, de Divittis $\mathrm{E}$, et al: Pure endoscopic endonasal odontoidectomy: anatomical study. Neurosurg Rev 30:189-194, 2007 


\section{O. Choudhri et al.}

27. Nayak JV, Gardner PA, Vescan AD, Carrau RL, Kassam AB, Snyderman CH: Experience with the expanded endonasal approach for resection of the odontoid process in rheumatoid disease. Am J Rhinol 21:601-606, 2007

28. Neo M, Asato R, Fujibayashi S, Ito H, Takemoto M, Nakamura $\mathrm{T}$ : Navigated anterior approach to the upper cervical spine after occipitocervical fusion. Spine (Phila Pa 1976) 34:E800-E805, 2009

29. Palmer JN, Kennedy DW: Historical perspective on imageguided sinus surgery. Otolaryngol Clin North Am 38:419428, 2005

30. Pollack IF, Welch W, Jacobs GB, Janecka IP: Frameless stereotactic guidance. An intraoperative adjunct in the transoral approach for ventral cervicomedullary junction decompression. Spine (Phila Pa 1976) 20:216-220, 1995

31. Roberts DW, Strohbehn JW, Hatch JF, Murray W, Kettenberger H: A frameless stereotaxic integration of computerized tomographic imaging and the operating microscope. J Neurosurg 65:545-549, 1986

32. Roessler K, Ungersboeck K, Dietrich W, Aichholzer M, Hittmeir K, Matula C, et al: Frameless stereotactic guided neurosurgery: clinical experience with an infrared based pointer device navigation system. Acta Neurochir (Wien) 139:551559, 1997

33. Rumboldt Z, Huda W, All JW: Review of portable CT with assessment of a dedicated head CT scanner. AJNR Am J Neuroradiol 30:1630-1636, 2009

34. Scholtes F, Signorelli F, McLaughlin N, Lavigne F, Bojanowski MW: Endoscopic endonasal resection of the odontoid process as a standalone decompressive procedure for basilar invagination in Chiari type I malformation. Minim Invasive Neurosurg 54:179-182, 2011

35. Sieśkiewicz A, Łysoń T, Mariak Z, Rogowski M: [Neuronavigation in transnasal endoscopic paranasal sinuses and cranial base surgery: comparison of the optical and electromagnetic systems.] Otolaryngol Pol 63:256-260, 2009 (Polish)
36. Spetzler RF, Hadley MN, Sonntag VKH: The transoral approach to the anterior superior cervical spine. A review of 29 cases. Acta Neurochir Suppl (Wien) 43:69-74, 1988

37. Ugur HC, Kahilogullari G, Attar A, Caglar S, Savas A, Egemen $\mathrm{N}$ : Neuronavigation-assisted transoral-transpharyngeal approach for basilar invagination - two case reports. Neurol Med Chir (Tokyo) 46:306-308, 2006

38. Veres R, Bagó A, Fedorcsák I: Early experiences with imageguided transoral surgery for the pathologies of the upper cervical spine. Spine (Phila Pa 1976) 26:1385-1388, 2001

39. Vougioukas VI, Hubbe U, Schipper J, Spetzger U: Navigated transoral approach to the cranial base and the craniocervical junction: technical note. Neurosurgery 52:247-251, 2003

40. Wolinsky JP, Sciubba DM, Suk I, Gokaslan ZL: Endoscopic image-guided odontoidectomy for decompression of basilar invagination via a standard anterior cervical approach. Technical note. J Neurosurg Spine 6:184-191, 2007

41. Wu JC, Huang WC, Cheng H, Liang ML, Ho CY, Wong TT, et al: Endoscopic transnasal transclival odontoidectomy: a new approach to decompression: technical case report. Neurosurgery 63 (1 Suppl 1):ONSE92-ONSE94, 2008

42. Yu Y, Wang X, Zhang X, Hu F, Gu Y, Xie T, et al: Endoscopic transnasal odontoidectomy to treat basilar invagination with congenital osseous malformations. Eur Spine J 22:1127-1136, 2013

Manuscript submitted November 18, 2013.

Accepted January 16, 2014.

Please include this information when citing this paper: DOI: 10.3171/2014.1.FOCUS13533.

Address correspondence to: Jayakar V. Nayak, M.D., Ph.D., Otolaryngology-Head \& Neck Surgery, Stanford University Medical Center, 801 Welch Rd., Stanford, CA 94305. email: jnayak@ohns. stanford.edu. 